\title{
Stabilizing effect of epoxidized sunflower oil as a secondary stabilizer for $\mathrm{Ca} / \mathrm{Hg}$ stabilized PVC
}

\author{
M. T. Taghizadeh*, N. Nalbandi, A. Bahadori \\ Department of Physical Chemistry, Faculty of Chemistry, University of Tabriz, Tabriz, Iran
}

Received 21 May; accepted in revised form 26 November 2007

\begin{abstract}
Unsaturated triglyceride oil sunflower was epoxidized and characterized by chemical and spectroscopic methods. Epoxidized sunflower oil (ESO) was used as an organic thermal co-stabilizer for rigid poly(vinyl chloride) (PVC) in the presence of tricalcium dicitrate $\left(\mathrm{Ca}_{3}\left(\mathrm{C}_{6} \mathrm{H}_{5} \mathrm{O}_{7}\right)_{2}\right)$ and mercury (II) acetate $\left(\mathrm{Hg}\left(\mathrm{CH}_{3} \mathrm{COO}\right)_{2}\right)$. The thermo-oxidative degradation of PVC was studied in the presence of these ternary stabilizer systems at 170, 180, 190 and $200^{\circ} \mathrm{C}$ in $\mathrm{N}_{2}$ atmosphere. The effects of metal carboxylate combination $\mathrm{Ca} / \mathrm{Hg}$ in the absence and in the presence of epoxidized sunflower oil on static heat treatment of PVC have been studied. The formation of polyene sequences was investigated by UV-visible and FT-IR spectroscopy and by comparing viscosity data obtained in the presence and in the absence of the additives. It was found that the additives retard the rate of degradation and reduce the extent of polymer chain scission associated with the thermal degradation of poly(vinyl chloride). Synergistic effects were found when stabilizer was blended in 50:50 weight ratios with either. It was found that ESO exerted a stabilizing effect on the degradation of PVC. The activation energy for degraded PVC in absence of stabilizers was $38.6 \mathrm{~kJ} \cdot \mathrm{mol}^{-1}$ and in the presence of $\mathrm{Ca} / \mathrm{Hg}$ and $\mathrm{Ca} / \mathrm{Hg} / \mathrm{ESO}$ were 53.3 and $64.7 \mathrm{~kJ} \cdot \mathrm{mol}^{-1}$ respectively.

In order of compare the efficiency of the epoxidized sunflower oil with these metal soap stabilizers, thermal stabilities were evaluated on the basis of evolved hydrogen chloride determined by conductometry technique and degree of discoloration are discussed.
\end{abstract}

Keywords: thermal properties, poly(vinyl chloride), thermal stabilization, thermo-oxidative degradation, secondary stabilizer

\section{Introduction}

Poly(vinyl chloride), PVC, is one of the leading thermoplastic materials. It stands second in the world after polyethylene so far as production is concerned. However, PVC shows low thermal stability [1]. It is generally accepted that poly(vinyl chloride), PVC, is an unstable polymer when exposed to high temperatures during its moldings and applications. Therefore, the poor thermal stability of PVC still remains one of its main problems [2]. Thermal degradation of PVC occurs by an autocatalytic dehydrochlorination reaction with the subsequent formation of conjugated double bonds [3].
Dehydrochlorination initiated at the labile site in the polymer chains. This leads to an extensive discoloration of the polymer and deterioration of its physical and mechanical properties. Possible defect structures in PVC are allylic chlorine [4], tertiary hydrogen and chlorine atom [5], end groups such as double bonds [6], oxygen containing group, peroxide residue [7], head-to-head structures [8]. Some authors have claimed that these structural irregularities are responsible and can account for the low thermal stability of PVC.

Although structural defects considerably increase the initial rate of PVC degradation, and indeed ini- 
tial rates of degradation at low conversions $(0.1 \pm 0.3 \%)$ have been shown to correlate well with allylic and/or tertiary chloride content of PVC [9]; it has been argued that on account of the low concentrations in normal PVC of these structural irregularities, that initiation of thermal degradation of PVC also takes place at regular monomer units [8]. In addition to the difficulties in identification and quantification of such small amount of labile allylic and tertiary chlorides within normal PVC structure, it is difficult to separate their effects on degradation from that of normal polymer units. It is now generally well accepted that random elimination of $\mathrm{HCl}$ from regular monomer sequence are responsible for the higher degree of conversion which degrades the polymer [10].

Because these changes are accompanied by deterioration on some of the useful properties of the polymer, it has become the practice to process PVC in the presence of heat stabilizers. Thermal stabilizers for PVC are known to function by replacing labile chlorine atoms in the polymer; they modify chain reactions and thereby inhibit the elimination of hydrogen chloride and interrupt the formation of polyene sequences in the polymer [5].

Additives that have found practical application as thermal stabilizers for PVC include metal soaps of carboxylic acids, organometallic compounds and inhibitors of radical chain reactions [9]. The most important stabilizers of PVC are different metal soaps like $\mathrm{Pb}, \mathrm{Cd}, \mathrm{Ba}, \mathrm{Ca}$ and $\mathrm{Zn}$ carboxylates and some di- and mono-alkyltin compounds, e.g., maleates, carboxylates, mercaptides [11]. A wide variety of stabilizers is used industrially to improve the thermal stability of the polymer. The commonly used stabilizers are usually basic lead salts that can react with the evolved hydrogen chloride gas thus they retard the deleterious catalytic action of the eliminated hydrogen chloride, or substances that can exchange the labile functional group in the backbone chain for other more stable substituent derived from the stabilizer; for example, metallic soaps [12], esters or mercaptides of alkyl tin or material whose stabilizing action is through intervention with the radical process of degradation such as quinone tin polymer.

Moreover, the stabilizer may function by disruption of the conjugated system, thus reducing the discoloration of the polymer. Mercaptans are typical example of these stabilizers. However, irrespective of their stabilizing efficiencies; they suffer from the deleterious effect of their byproduct, mostly metal chloride, accumulated during the reaction of these stabilizers with the polymeric chain [13].

These metal chlorides are considered as strong catalysts for the subsequent dehydrochlorination process, and they are responsible for the sudden blackening of certain formulation, and may present a serious environmental problem. This has led, recently, to the extensive use of stabilizers of an organic nature for the thermal stabilization of PVC [14].

Epoxy compounds are well known as typical nonmetallic stabilizers for PVC [15]. They are generally regarded as secondary stabilizers used to enhance the effectiveness of metal soaps. They act as acceptors for the liberated hydrogen chloride $[16,17]$ and retardants for the appearance of discoloration $[18,19]$. The effects of epoxidized sunflower (ESO) on the thermal degradation and stabilization of PVC in the presence of metal carboxylates $(\mathrm{Ba} / \mathrm{Cd}$ and $\mathrm{Ca} / \mathrm{Zn}$ stearates) have been investigated. ESO showed excellent properties as secondary stabilizer for PVC [20].

In this work, commercial sunflower oil was epoxidized. The stabilizing effect of epoxidized sunflower oil (ESO) on the thermal degradation of $\mathrm{PVC}$ in presence and absence of mixture tricalcium dicitrate and mercury (II) acetate and the ratios of $\mathrm{Ca}$ and $\mathrm{Hg}$ carboxylate $(1 / 1,1 / 2,1 / 3,2 / 1,2 / 2,2 / 3$, $3 / 1,3 / 2)$ and $\mathrm{ESO}$ in combination with $\mathrm{Ca} / \mathrm{Hg}$ carboxylate under atmosphere of nitrogen have been investigated. The dehydrochlorination process in PVC degradation has been studied in detail using viscometric and spectrophotometric analysis and the values of the kinetic parameters have been calculated.

\section{Experimental}

\subsection{Materials}

Poly(vinyl chloride), PVC, suspension, with $K$ value of 69-71; tricalcium dicitrate and mercury (II) acetate from Fluka Company were used for this investigation. PVC was purified by solution in THF/acetone mixture and precipitated with constant stirring in a large excess of methanol. The precipitated polymer was filtered off after $24 \mathrm{~h}$, 
washed with methanol, and air-dried $\left(60^{\circ} \mathrm{C}\right)$ [21]. Commercial sunflower oil, was used. Epoxdized sunflower oil was prepared by epoxidation. Amberlite IR-120 cross-linked ploy(styrene-sulfonic acid) ion exchange resin and hydrogen peroxide (30\%) were purchased from Fluka Company. All analytical grade solvents were used as obtained from prolabo.

\subsection{Preparation of epoxidized sunflower oil (ESO)}

The epoxidation of sunflower oil was carried out at $50^{\circ} \mathrm{C}$ using peroxy-acetic acid prepared in situ by reacting hydrogen peroxide $(30 \% \mathrm{v} / \mathrm{v})$ with excess glacial acetic acid and Amberlite IR-120 [22]. The level of epoxidation was determined using a standard analytical method [23]. ESO with oxirane oxygen level $4.5 \%$ was prepared.

\subsection{Degradation of PVC: rates of dehydrochlorination}

Degradation studies were carried out using PVC powder in the presence of ESO $(0.04 \mathrm{~g})$, tri-calcium dicitrate and mercury (II) acetate $(0.008 \mathrm{~g})$ at 170 , 180,190 and $200^{\circ} \mathrm{C}$ under nitrogen atmosphere. The PVC sample $(0.5 \mathrm{~g})$ was mixed thoroughly with an appropriate amount of the additive and transferred into a degradation tube. The tube was connected to a source of nitrogen maintained at a flow rate of $250 \mathrm{ml} \cdot \mathrm{min}^{-1}$. The degradation tube was then immersed in a thermostat oil bath. The amount of evolved $\mathrm{HCl}$ was established after various periods of time by conductometry. The extent of dehydrochlorination (conversion \%) was calculated from the ratio of evolved $\mathrm{HCl}$ to the amount available in the polymer [24].

\subsection{UV-visible analysis}

UV-visible spectra of purified PVC solutions in distilled 1,2-dichloroethane (2 g/l) were obtained by using a Shimadzu 120-02 UV-visible spectrophotometer. The polymer was purified by dissolution in 1,2-dichlroethane, precipitation with methanol and filtration.

The extent of discoloration of the degradation polymer sample was measured colorimetrically at $\lambda=$ $360 \mathrm{~nm}$ as a function of the degradation time.

\subsection{FT-IR analysis}

IR spectra were measured using a Shimadzu infrared spectrophotometer (FTIR-4300) in the range $600-4000 \mathrm{~cm}^{-1}$ at $25^{\circ} \mathrm{C}$. All the samples were mixed with $\mathrm{KBr}$ for these analyses.

\subsection{Intrinsic viscosity measurements}

The degraded PVC $(0.05 \mathrm{~g})$ was purified by solution in cyclohexanone and precipitated in a large excess of methanol. The precipitated polymer was filtered off, washed several times with methanol, dried in vacuum at room temperature. Intrinsic viscosities were determined using a setgvis kinematic from measurements in cyclohexanone solution at $30^{\circ} \mathrm{C}$. The intrinsic viscosities and relative and specific viscosities of solutions were calculated by Equations (1)-(3) [25]:

$$
\begin{aligned}
& \eta=\frac{\left(\eta_{s p}+3 \ln \eta_{r e l}\right)}{4 C} \\
& \eta_{r e l}=\frac{\eta}{\eta_{0}}=\frac{t}{t_{0}} \\
& \eta_{s p}=\eta_{r e l}-1
\end{aligned}
$$

where $\eta, \eta_{s p}$ and $\eta_{r e l}$ are the intrinsic, specific and relative viscosities, respectively, $C$ is the concentration of the solution $[\mathrm{g} / \mathrm{ml}]$. In these expressions, $t$ is the time of flow of polymer solution and $t_{0}$, the time of flow of solvent at the temperature of measurement. The ratios of the intrinsic viscosity of PVC samples degraded in the presence of additives to the intrinsic viscosity of undegraded samples, $\left(\eta / \eta_{0}\right)$, was used to deduce the effect of the additives on the degradation of PVC.

\section{Results and discussion}

\subsection{Effect of the stabilizer concentration on the efficiency of stabilization}

The results of the dehydrochlorination of rigid PVC $(0.5 \mathrm{~g})$ stabilized by tricalcium dicitrate in different concentrations $(0.4-3 \%$ w/w) are shown in Figure 1 . They indicate that with increasing of [tricalcium dicitrate]/[PVC] ratio up to about $1.6 \% \mathrm{w} / \mathrm{w}$ of stabilizer, the rate of dehydrochlorination decreases and thereafter it increases. It can be seen from the results that the effect of $\mathrm{Ca}$ on the degra- 


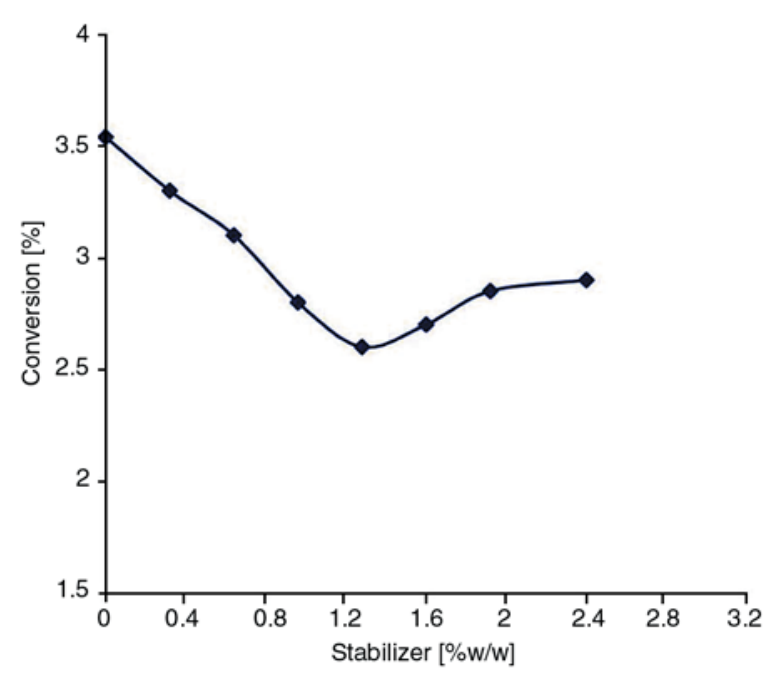

Figure 1. Conversion [\%] of rigid PVC at $180^{\circ} \mathrm{C}$ with $0.5 \mathrm{~g}$ of PVC for various concentration of tricalcium dicitrate

dation of PVC is not additive: showing antagonistic effect up to $1.6 \% \mathrm{w} / \mathrm{w} \mathrm{Ca}$ and synergistic effect at higher $\mathrm{Ca}$. The data of others compounds show that the stabilizing efficiency of mercury (II) acetate and ESO is greater with concentration of $1.6 \% \mathrm{w} / \mathrm{w}$ and $0.8 \% \mathrm{w} / \mathrm{w}$, respectively. It is known that labile chlorine atoms in PVC, especially allylic and tertiary chlorine, reduce the thermal stability of this polymer [26-31].

\subsection{Stabilization of thermally degraded rigid $\mathrm{PVC}$ using mixed stabilizer $(\mathrm{Ca} / \mathrm{Hg})$ in the absence of ESO}

The results of the dehydrochlorination of thermally degraded rigid PVC $(0.5 \mathrm{~g})$ at $180^{\circ} \mathrm{C}$ in $\mathrm{N}_{2}$ in the presence of the mixed stabilizers $(\mathrm{Ca} / \mathrm{Hg})$ are shown in Figure 2. The results clearly reveal the greater stabilizing efficiency of the mixed stabilizers, reaching its maximum when the two stabilizers were mixed in a 2:2 ratio, respectively. It can be seen that the stabilizing effect of $\mathrm{Ca}$ stabilizer is enhanced by the presence of $\mathrm{Hg}$ stabilizer. The marked reductions in the rate of dehydrochlorination using mixed stabilizer $(\mathrm{Ca} / \mathrm{Hg})$ may be attributed to synergism of $\mathrm{Hg}$ and $\mathrm{Ca}$ compounds in stabilizing PVC against thermal degradation.

The mechanism by which metal soaps stabilize PVC against thermal degradation was first proposed by Frye and Horst [32, 33] and it involves the replacement of labile chlorine atoms within PVC

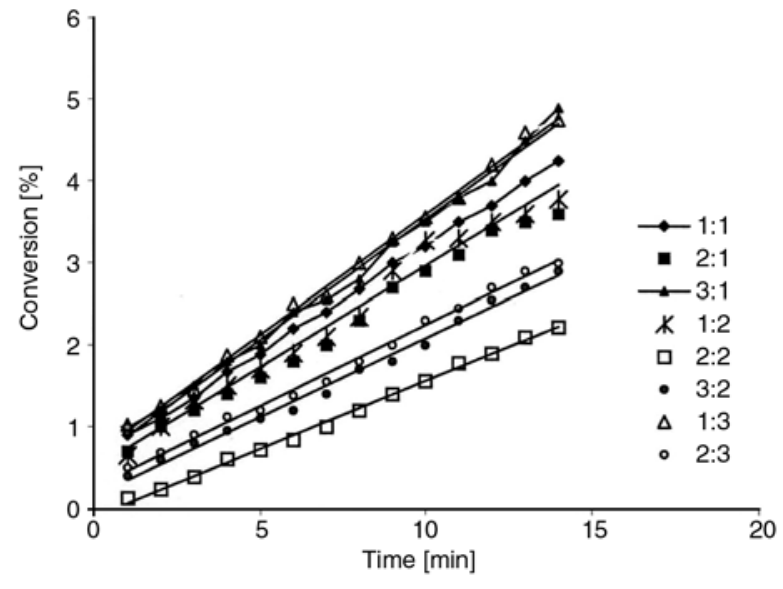

Figure 2. Conversion [\%] of rigid PVC at $180^{\circ} \mathrm{C}$ with $0.5 \mathrm{~g}$ of PVC containing various ratios of $\mathrm{Ca} / \mathrm{Hg}$ in the absence of ESO

structure with carboxylate groups which are more stable to heat treatment.

\subsection{Stabilization of thermally degraded rigid PVC using mixed stabilizer (Ca/Hg/ESO)}

It is well known that the ability to prevent dehyderochlorination of PVC is an important characteristic in the evaluation of the stabilizing effect. The experimental values of dehydrochlorination of rigid $\mathrm{PVC}$ at $180^{\circ} \mathrm{C}$ in $\mathrm{N}_{2}$ in the presence of additives and mixed additives are given in Figure 3.

Figure 3 shows the dehydrochlorination curves of PVC containing metal carboxylates and $\mathrm{Ca} / \mathrm{Hg}$ $(2 / 2)$ in the absence and in the presence of ESO for comparable the $\mathrm{Ca} / \mathrm{Hg}$ stabilizer in the weight ratio of $1 / 1$ when used in combination with ESO was found to be most effective.

It can be seen from Figure 3 that the lower value was obtained for the $\mathrm{Ca} / \mathrm{Hg}(1 / 1)$-ESO, for this synergetic mixture of stabilizers, the presence of ESO reduced effectiveness the initial rates of dehydrochlorination (DHC).

The investigated ESO exhibits a greater stabilizing efficiency compared to others. The greater stabilizing efficiency is illustrated not only by lower rates of DHC during the subsequent stages of degradation reaction, but also by the longer induction periods or the thermal stability value $\left(T_{s}\right)$ during which no detectable amounts of hydrogen chloride gas are liberated and the values of the required time for dehydrochlorination to attain $1 \%$ conversion, $t_{D H}$, for all additives are shown in Table 1. 
Table 1 gives the values of induction times and of initial rates of DHC which were computed from the slopes of the kinetic curves (Figure 3), The addition of $\mathrm{ESO}$ to $\mathrm{Ca} / \mathrm{Hg}(1 / 1)$ increases the induction time and decreases the rate of DHC.

The effectiveness of ESO depends on the amount of epoxy groups in the molecule. The higher the amount of epoxy groups the better is the effectiveness. This stabilizing effect of ESO on the thermal degradation of PVC is a result of the facile reaction of $\mathrm{HCl}$ with epoxy groups to form chlorohydrins which is thought to reduce the autocatalytic effect of the $\mathrm{HCl}$ evolved. Furthermore, the esterification and etherification reactions which occur with unstable allylic chlorine groups in PVC provide an explanation for the very low values of the initial rates of DHC observed when ESO is used in combination with the $\mathrm{Ca} / \mathrm{Hg}$ stearates. The observed enhanced stabilization effectiveness of the metal soaps of ESO is considered to results from the combined action of the peroxide [34].

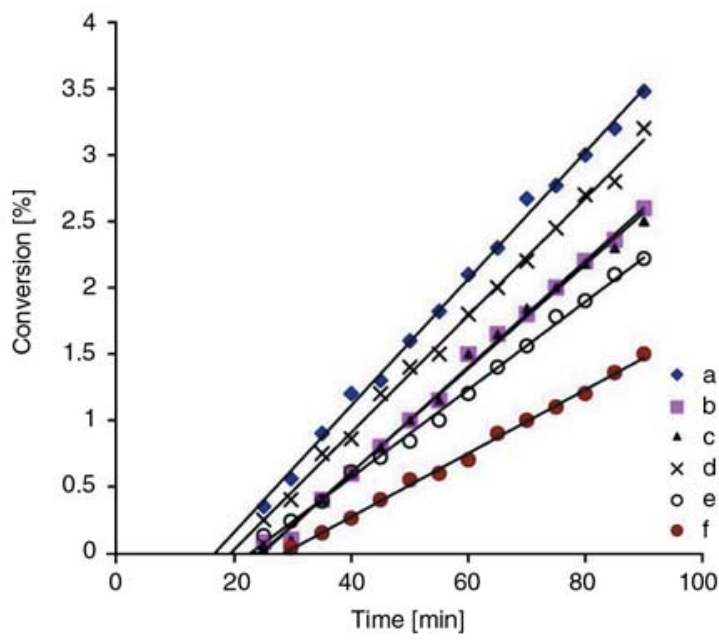

Figure 3. Comparison of stabilizers on the rate of dehydrochlorination of degraded $\mathrm{PVC}$ at $180^{\circ} \mathrm{C}$ : (a) PVC alone, (b) PVC with $0.008 \mathrm{~g} \mathrm{Hg}$ stabilizer, (c) PVC with $0.008 \mathrm{~g}$ Ca stabilizer, (d) PVC with $0.04 \mathrm{~g}$ ESO stabilizer (e) $\mathrm{PVC}$ with $\mathrm{Ca} / \mathrm{Hg}$ stabilizers, (f) PVC with $\mathrm{Ca} / \mathrm{Hg} / \mathrm{ESO}$ stabilizer

Table 1. Dehydrochlorination data at $180^{\circ} \mathrm{C}$ with $0.5 \mathrm{~g}$ PVC containing various stabilizers systems

\begin{tabular}{|l|c|c|c|}
\hline $\begin{array}{c}\text { Stabilizer } \\
\text { system }\end{array}$ & $\begin{array}{c}\mathbf{T}_{\mathbf{s}} \\
{[\mathbf{m i n}]}\end{array}$ & $\begin{array}{c}\mathbf{t}_{\mathbf{D H}} \\
{[\mathbf{m i n}]}\end{array}$ & $\begin{array}{c}\mathbf{R} \cdot \mathbf{1 0}^{\mathbf{2}} \\
{[\text { conversion\%/min] }}\end{array}$ \\
\hline none & 14 & 38 & 4.8 \\
\hline $\mathrm{Ca} / \mathrm{Hg}$ & 23 & 53 & 3.3 \\
\hline $\mathrm{Cu} / \mathrm{Hg} / \mathrm{ESO}$ & 38 & 71 & 2.4 \\
\hline
\end{tabular}

\subsection{The UV-visible absorption spectra of PVC samples degraded in $\mathrm{N} 2$ at $180^{\circ} \mathrm{C}$}

After the degradation of PVC in inert atmosphere, the resulting material was dissolved in freshly distilled 1.2-dichloroethane and UV-visible spectrum was recorded. The combination of $\mathrm{Ca} / \mathrm{Hg}$, reduced considerably the initial rates of DHC. The lower value was obtained for the $\mathrm{Ca} / \mathrm{Hg}$. For this synergetic mixture of stabilizers, UV-visible characterization of purified PVC samples treated for various times at $180^{\circ} \mathrm{C}$ and heated $60 \mathrm{~min}$ at $180^{\circ} \mathrm{C}$ for combination of $\mathrm{Ca} / \mathrm{Hg}$ were realized. As shown in Figures 4 and 5, the combined effect would reduce the amounts of $\mathrm{HCl}$ evolved leading to the formation of short polyene sequences absorbing in the ultraviolet region.

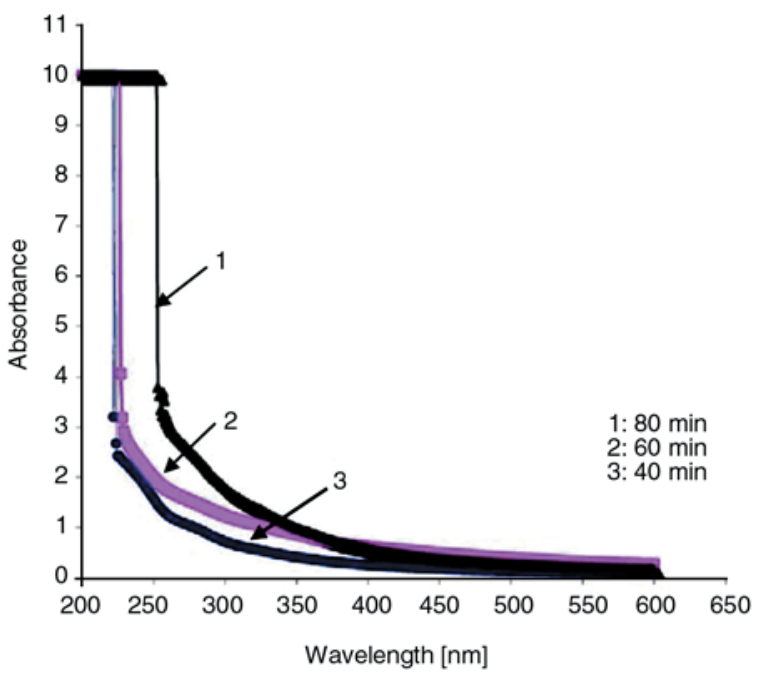

Figure 4. UV-visible spectra of purified PVC stabilized with $\mathrm{Ca} / \mathrm{Hg}(1 / 1)$ and treated for various times at $180^{\circ} \mathrm{C}$

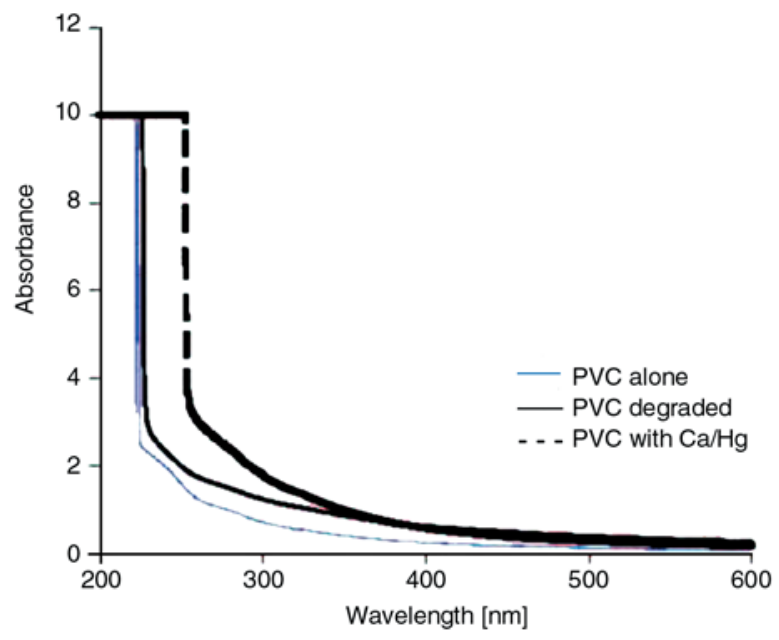

Figure 5. UV-visible spectra of purified PVC stabilized with $\mathrm{Ca} / \mathrm{Hg}$ heated $60 \mathrm{~min}$ at $180^{\circ} \mathrm{C}$ 


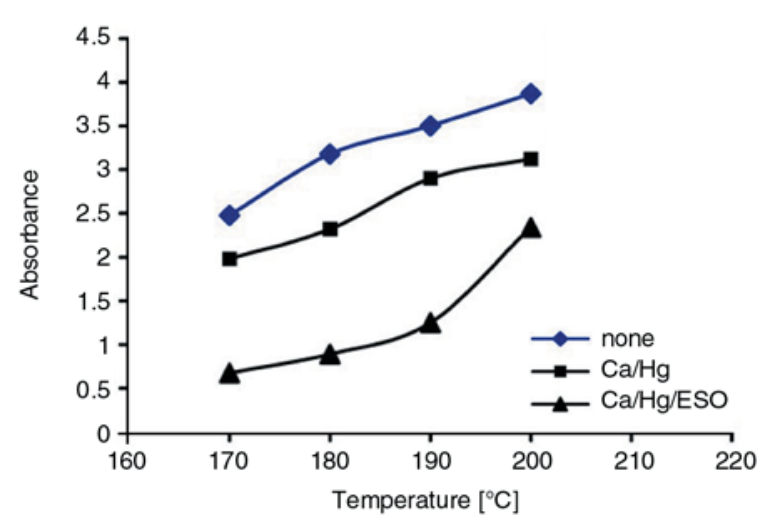

Figure 6. Absorbance-temperature curves for PVC thermally degraded at 90 min in $\mathrm{N}_{2}$ (samples concentration, $50 \mathrm{mg}$ in $25 \mathrm{ml}$ 1,2-dichloroethane)

The absorption bands were characterized using the maximum wavelength values [35]. The absorption pattern indicates that longer conjugated bond systems are present in PVC samples degraded in the absence of these materials than in the PVC samples degraded in the presence of these materials. The UV-visible absorption spectra of PVC samples degraded in $\mathrm{N}_{2}$ are generally regarded as not providing accurate or reliable information on the level of unstauration in the polymer, however such spectra could be useful in providing a basis for assessing the relative effectiveness of stabilizers in stabilizing PVC against degradation.

A proof for the high stabilizing efficiency is obtained by measuring the extent of discoloration of degraded PVC. The extent of coloration was determined colorimetrically by measuring the absorbance at $\lambda=360 \mathrm{~nm}$ for the various samples as a function of the degradation temperatures. The results shown in Figure 6, reveal the lower discoloration of PVC samples was obtained for the $\mathrm{Ca} / \mathrm{Hg}(1 / 1)$-ESO.

\subsection{FT-IR spectra of degraded PVC samples}

In order to examine the effect of stabilizer on PVC degradation by FT-IR spectroscopy, films of appropriate thickness to give adequate absorption in the required range were prepared. The FT-IR spectra of undegraded PVC and unstabilized degraded PVC and treated for various times are shown in Figure 7. In this study attention was focused on change in the absorption pattern in three main regions: (a) stretching vibration of the $\mathrm{COO}^{-}$group between 1500 and $1600 \mathrm{~cm}^{-1}$; (b) stretching vibration of the

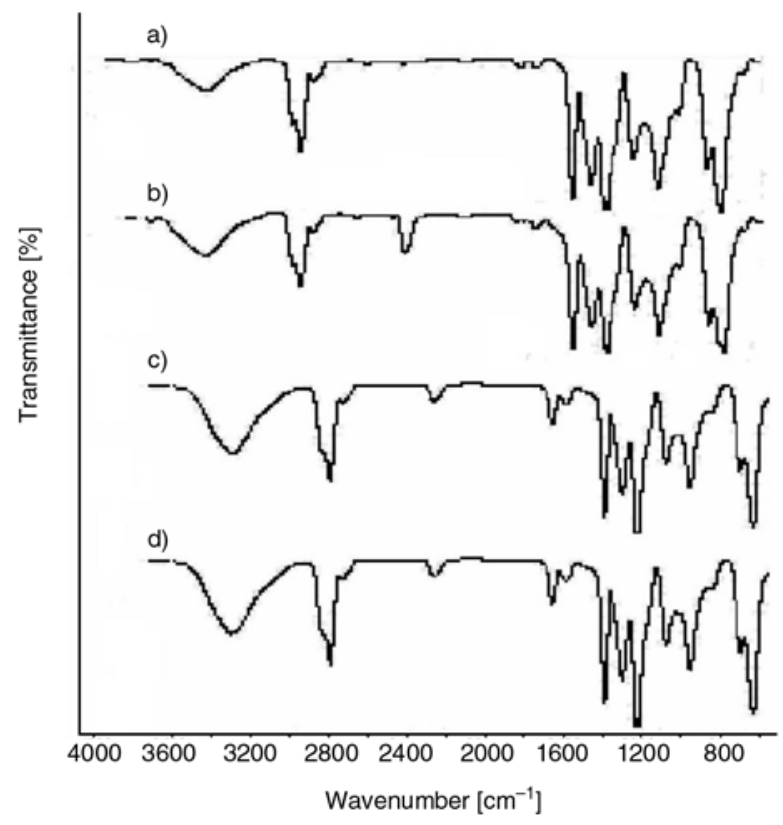

Figure 7. Variations in the FT-IR spectra of PVC samples undegraded and and degraded at $170^{\circ} \mathrm{C}$ in $\mathrm{N}_{2}$ as a function of degradation time: (a) undegraded PVC, (b) heated $30 \mathrm{~min}$, (c) heated $60 \mathrm{~min}$, (d) heated $90 \mathrm{~min}$

$\mathrm{C}=\mathrm{C}$ group between 1600 and $1680 \mathrm{~cm}^{-1}$ and (c) the $\mathrm{C}=\mathrm{O}$ stretching vibration between 1690 and $1750 \mathrm{~cm}^{-1}$.

The FT-IR absorption pattern characteristic of alkenic structure was observed for all the degraded PVC samples at about $1630 \mathrm{~cm}^{-1}$. It indicated that when the temperature increases, absorption by $\mathrm{C}=\mathrm{C}$ would increase, although the absorption by $\mathrm{C}=\mathrm{O}$ group has been increased. The assignment of the observed band to the stretching vibration of the $\mathrm{C}=\mathrm{C}$ bonds in PVC is favored by the fact that a rather weak absorption band at $1710 \mathrm{~cm}^{-1}$ attributed to $\mathrm{C}=\mathrm{O}$ was observed for all PVC samples. The spectral range $1690-1750 \mathrm{~cm}^{-1}$ is characteristic of absorption bands of esters, carboxylic acids and ketones, and is indicative of the esterification of the polymer chain.

FT-IR spectra of PVC alone and PVC stabilized with $\mathrm{Ca} / \mathrm{Hg}(1 / 1)$ in the absence and in presence of ESO are presented in Figure 8.

In comparison with PVC alone, stabilized PVC samples show a band at $1560 \mathrm{~cm}^{-1}$ is due to the metal carboxylate which has not yet been mentioned. The band at $1730 \mathrm{~cm}^{-1}$ is due to oxidation products [36]. 


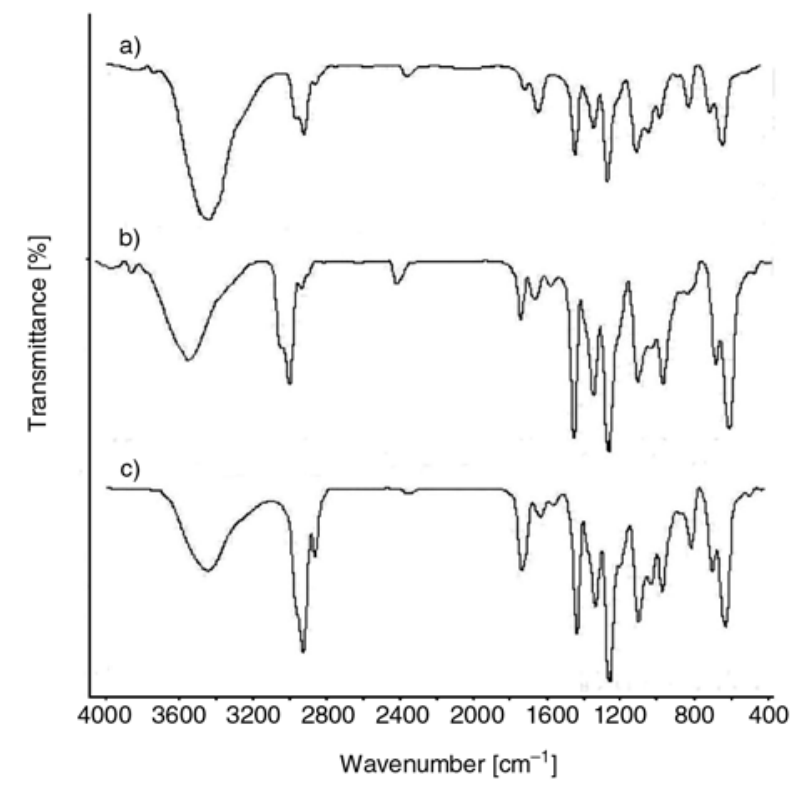

Figure 8. FT-IR spectra of (a) PVC alone, (b) PVC stabilized with $\mathrm{Ca} / \mathrm{Hg}(1 / 1)$ and heated $90 \mathrm{~min}$ at $190^{\circ} \mathrm{C}$, (c) PVC stabilized with $\mathrm{Ca} / \mathrm{Hg}$ (1/1)-ESO and heated $90 \mathrm{~min}$ at $190^{\circ} \mathrm{C}$

Figure 8 shows the FT-IR spectra of unstabilzed $\mathrm{PVC}$ and $\mathrm{PVC}$ stabilized with $\mathrm{Ca} / \mathrm{Hg}$ and $\mathrm{PVC}$ stabilized with $\mathrm{Ca} / \mathrm{Hg} / \mathrm{ESO}$. The FT-IR spectra of PVC containing $\mathrm{Ca} / \mathrm{Hg} / \mathrm{ESO}$ characterized by an absorption band in the $1710-1730 \mathrm{~cm}^{-1}$ range. This band was absent from the spectrum of unstabilized PVC. When combined with the results from the FT-IR studies on the interaction of $\mathrm{PVC}$ with $\mathrm{Ca} / \mathrm{Hg}$ and with $\mathrm{Ca} / \mathrm{Hg} / \mathrm{ESO}$, these results show that these stabilizers stabilize PVC against thermal degradation by replacing labile chlorine atoms in PVC in addition to acting as $\mathrm{HCl}$ scavenger.

From the aforementioned observation and results, it seems reasonable to propose the following mechanism for the stabilization of thermally degraded $\mathrm{PVC}$ in the presence of $\mathrm{Ca} / \mathrm{Hg} / \mathrm{ESO}$ : The esterification and reactions which occur with allylic chlorine groups in PVC provide an explanation for the synergism observed in the stabilization of PVC containing a combination of an epoxy compound with metal stabilizer [37]. The mechanism of epoxidation can be displayed by Equations (4), (5) and (6)):

$$
\begin{aligned}
& 2-\mathrm{CH}_{2}(-\mathrm{CH}=\mathrm{CH}-\mathrm{CHCl})_{n}-\mathrm{CH}_{2}-\mathrm{CH}_{3}+ \\
& n \mathrm{Ca}_{3}\left(\mathrm{O}_{2} \mathrm{CR}\right)_{2} \rightarrow \mathrm{NO} \text { reaction }
\end{aligned}
$$

$2-\mathrm{CH}_{2}(-\mathrm{CH}=\mathrm{CH}-\mathrm{CHCl})_{n}-\mathrm{CH}_{2}-\mathrm{CH}_{3}+n \mathrm{Hg}(\mathrm{OAC})_{2}$ $\rightarrow 2-\mathrm{CH}_{2}-(\mathrm{CH}=\mathrm{CH}-\mathrm{CH}(\mathrm{OAC}))_{n}-\mathrm{CH}_{2}-\mathrm{CH}_{3}+$ $n \mathrm{HgCl}_{2}$

$$
\begin{aligned}
& 2-\mathrm{CH}_{2}(-\mathrm{CH}=\mathrm{CH}-\mathrm{CHCl})_{n}-\mathrm{CH}_{2}-\mathrm{CH}_{3}+ \\
& n \mathrm{Ca}_{3}\left(\mathrm{O}_{2} \mathrm{CR}\right)_{2}+n \mathrm{Hg}(\mathrm{OAC})_{2} \rightarrow \\
& \left.n-\mathrm{CH}_{2}-(\mathrm{CH})=\mathrm{CH}-\mathrm{CH}(\mathrm{OAC})\right)_{n}-\mathrm{CH}_{2}-\mathrm{CH}_{3}+ \\
& \mathrm{Hg}\left(\mathrm{O}_{2} \mathrm{CR}\right)_{2}+\mathrm{CaCl}_{2}
\end{aligned}
$$

Epoxy compounds are well known as typical nonmetallic stabilizers for PVC [20]. They are generally regarded as secondary stabilizers used to enhance the effectiveness of metal soaps. They act as acceptors for the liberated hydrogen chloride [9, 21] and retardants for the appearance of discoloration (as in Equations (7) and (8)):

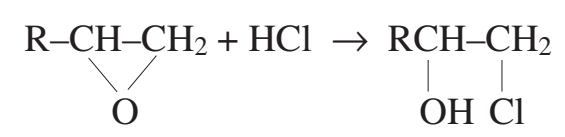<smiles>[Z6]OCC(O)C(O)CCC[PH2+]C1OC1[R]</smiles>

\subsection{Extent of thermal degradation: intrinsic viscosity}

The variation in the values of relative intrinsic viscosities $\eta / \eta_{0}$; where $\eta_{0}$ is the intrinsic viscosity of undegraded polymer; and $\eta$ is the intrinsic viscosity of the degraded PVC samples with degradation time are shown in Figure 9. The major types of reactions that may take place during the thermal degradation of PVC in $\mathrm{N}_{2}$ at moderate temperature such as dehydrochlorination, polymer chain scission, cross- linking, would influence the viscosity of the degraded polymer samples. It has been reported that the intrinsic viscosity of PVC under-

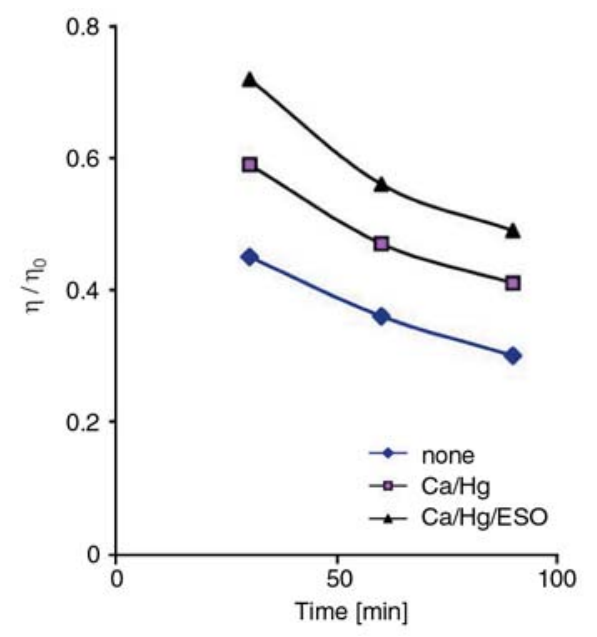

Figure 9. Relative intrinsic viscosity for PVC degraded in $\mathrm{N}_{2}$ at $180^{\circ} \mathrm{C}$ after $90 \mathrm{~min}$ 
going degradation decreases initially to a minimum and then increases with further increase in the number of double bonds in the polymer [38], probably due to reduced flexibility along the main polymer chain. Therefore, in low extents of degradation, it can be assumed that reactions accompanying dehydrochlorination are negligible and that the values of intrinsic viscosity would represent the extent of degradation, the lower the values of intrinsic viscosity, the greater the extent of degradation. The results in Figure 9 show that the values of intrinsic viscosity obtained for PVC samples degraded in the presence of stabilizers are much higher than the values for unstabilized PVC samples. These results are indicative of a stabilizing effect of the materials on the thermal degradation of PVC.

\subsection{Kinetics of dehydrochlorination of PVC}

\subsubsection{Determination of reaction order with respect of stabilizer at constant amount of PVC}

The rate of reaction is determined in Equation (9):

$R=\frac{\mathrm{d} x}{\mathrm{~d} t}$

where $R$ is the rate of the reaction and $x$ is the concentration of product in the reaction.

The plots of conversion \% vs. [S] are linear and slopes of the lines give the rate of the reaction dehydrochlorination. The rate of dehydrochlorina-

Table 2. Effect of metal carboxylate amount on the rate of dehydrochlorination of $\mathrm{PVC}$ at $180^{\circ} \mathrm{C}$ with $0.5 \mathrm{~g}$ polymer

\begin{tabular}{|c|c|c|}
\hline \multirow{2}{*}[\mathbf{S}]{$/ \mathbf{g}$} & \multicolumn{2}{|c|}{$\mathbf{R} \cdot \mathbf{1 0}^{2}[$ conversion\%/min] } \\
\cline { 2 - 3 } & $\mathbf{H g}\left(\mathbf{C H}_{\mathbf{3}} \mathbf{C O O}\right)_{\mathbf{2}}$ & $\mathbf{C a}_{3}\left(\mathbf{C}_{\mathbf{6}} \mathbf{H}_{\mathbf{5}} \mathbf{O}_{7}\right)_{\mathbf{2}}$ \\
\hline 0.002 & 4.7 & 4.5 \\
\hline 0.004 & 4.4 & 4.3 \\
\hline 0.006 & 4.1 & 4.2 \\
\hline 0.008 & 4.0 & 3.9 \\
\hline
\end{tabular}

Table 3. Effect of ESO amount on the rate of dehydrochlorination of $\mathrm{PVC}$ at $180^{\circ} \mathrm{C}$ with $0.5 \mathrm{~g} \mathrm{PVC}$

\begin{tabular}{|c|c|}
\hline$[\mathbf{E S O}] / \mathbf{g}$ & $\mathbf{R} \cdot \mathbf{1 0}^{\mathbf{2}}[$ conversion \%/min] \\
\hline 0.01 & 0.7 \\
\hline 0.02 & 4.6 \\
\hline 0.03 & 4.4 \\
\hline 0.04 & 4.2 \\
\hline
\end{tabular}

tion depends on the concentration of stabilizer and polymer as in Equations (10) and (11):

$R=k \cdot[\mathrm{PVC}]^{\alpha}[\mathrm{S}]^{\beta}$

$R=k^{\prime}[\mathrm{S}]^{\beta}$

Experimental values of dehydrochlorination of all additives are given in Tables 2 and 3 and depicted in Figures 10, 11 and 12.

The plot of $\ln R v s . \ln [\mathrm{S}]$ is linear and for $\mathrm{Hg}, \mathrm{Ca}$ and ESO stabilizers is plotted in Figure 13. The slopes of the lines give the order of the reaction with respect to stabilizers.

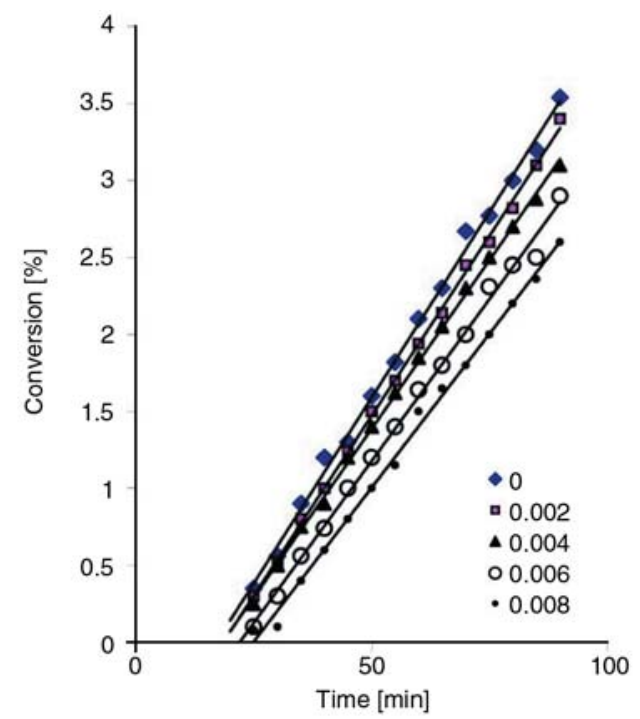

Figure 10. Conversion [\%] of PVC at $180^{\circ} \mathrm{C}$ with $0.5 \mathrm{~g}$ of PVC for various amounts of mercury (II) acetate $[\mathrm{g}]$

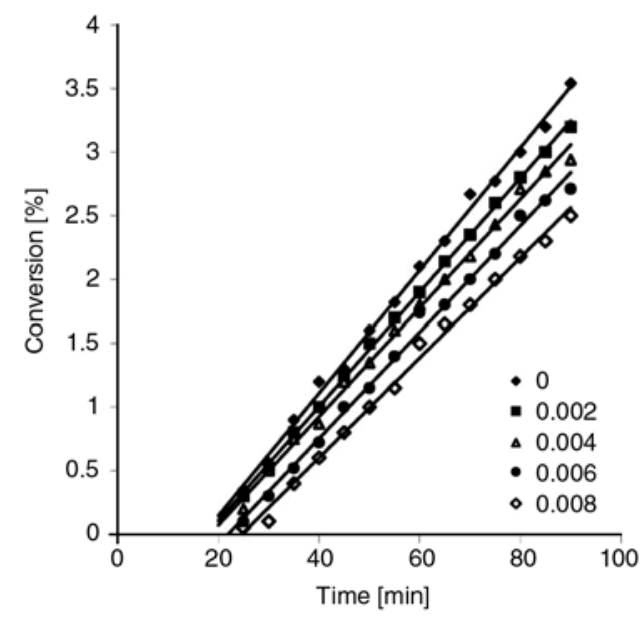

Figure 11. Conversion [\%] of $\mathrm{PVC}$ at $180^{\circ} \mathrm{C}$ with $0.5 \mathrm{~g}$ of $\mathrm{PVC}$ for various amounts of tricalcium dicitrate $[\mathrm{g}]$ 


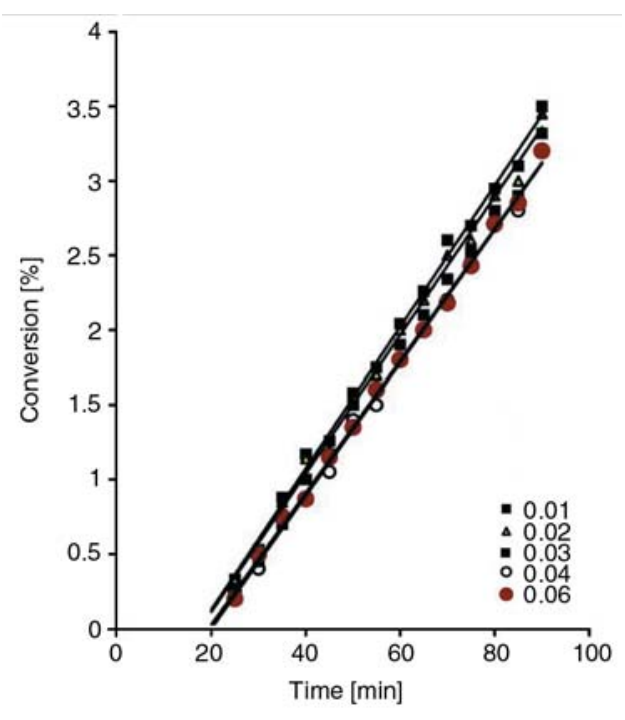

Figure 12. Conversion [\%] of $\mathrm{PVC}$ at $180^{\circ} \mathrm{C}$ with $0.5 \mathrm{~g}$ of PVC for various amounts of epoxidized sunflower oil $[\mathrm{g}]$

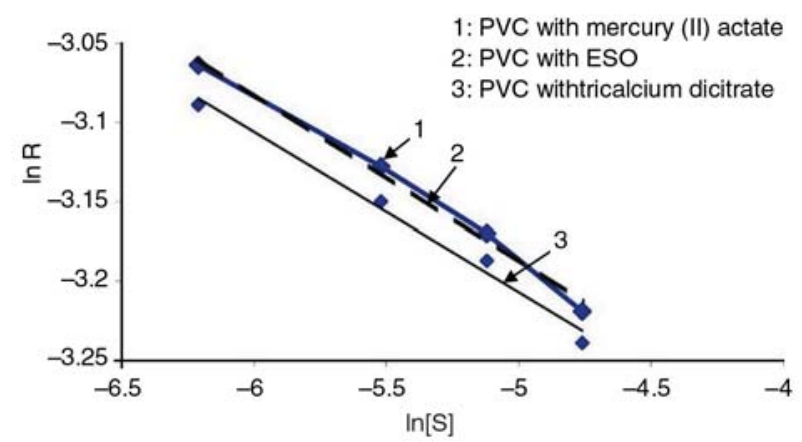

Figure 13. $\ln R$ vs. $\ln [\mathrm{S}]$ for mercury (II) acetate, tricalcium dicitrate and $\mathrm{ESO}$ stabilizer at $180^{\circ} \mathrm{C}$

From the data of Tables 2 and 3 the reaction order with respect to mercury (II) acetate, tricalcium dicitrate and ESO were determined $-1.05,-1.01$ and -0.05 respectively. These results show that all stabilizers are the negative orders. Thus the more negative reaction order shows that the more PVC stabilization. It is clear that $\mathrm{Hg}\left(\mathrm{CH}_{3} \mathrm{COO}\right)_{2}$ and $\mathrm{Ca}_{3}\left(\mathrm{C}_{6} \mathrm{H}_{5} \mathrm{O}_{7}\right)_{2}$ exhibited a greater stabilizing efficiency relative to ESO.

\subsubsection{Temperature effect on the rate dehydrochlorination in degradation of PVC}

The rates of degradation of $\mathrm{PVC}$ at various temperatures are shown in Figure 14. It can be seen that the extent of dehydrochlorination at temperatures between $170-200^{\circ} \mathrm{C}$ is linear.

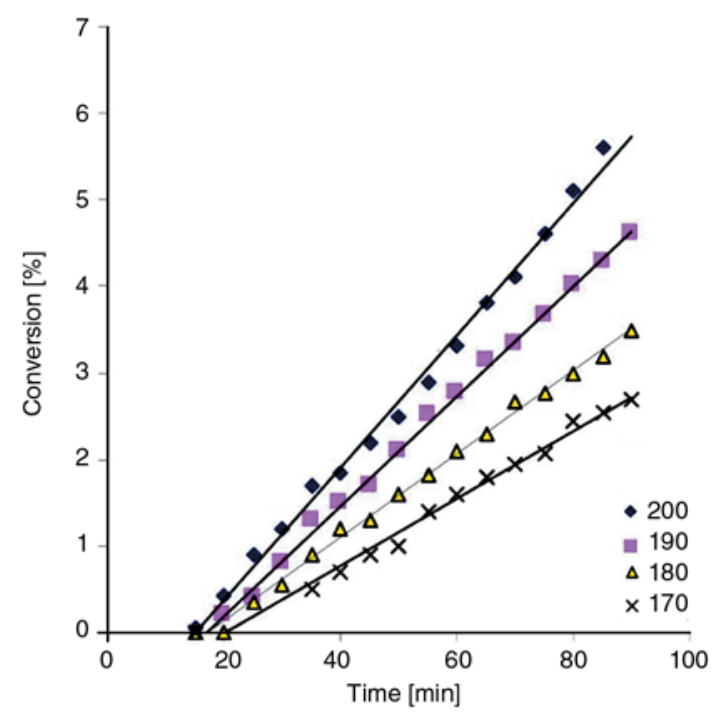

Figure 14. Conversion [\%] of PVC with $0.5 \mathrm{~g}$ of PVC at different temperatures

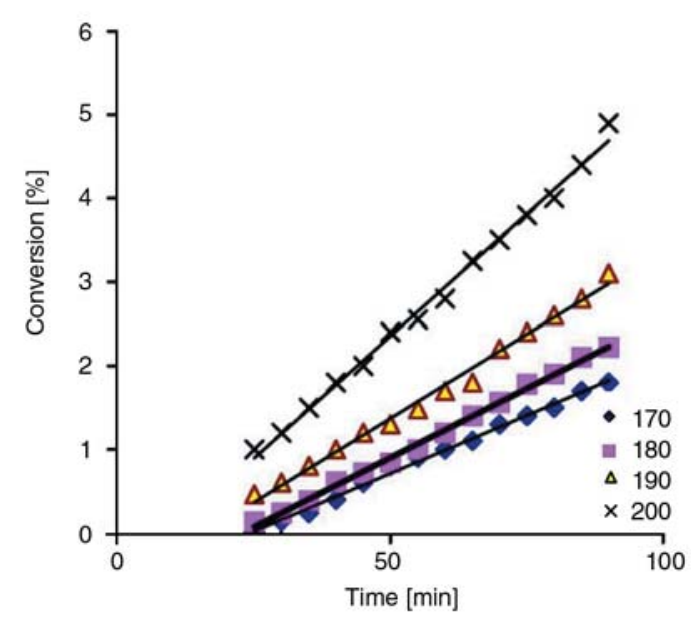

Figure 15. Conversion [\%] of PVC with $0.5 \mathrm{~g}$ of $\mathrm{PVC}$ in the presence of $\mathrm{Hg} / \mathrm{Ca}(2 / 2)$ at different temperatures

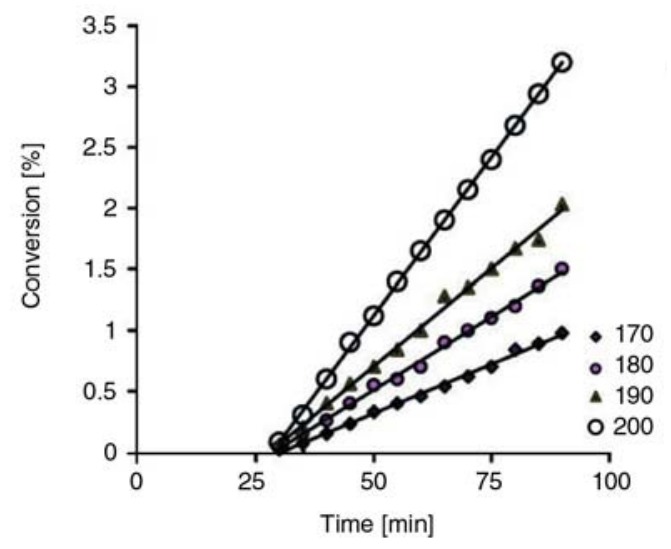

Figure16. Conversion [\%] of PVC with $0.5 \mathrm{~g}$ of $\mathrm{PVC}$ in the presence of $\mathrm{Hg} / \mathrm{Ca} / \mathrm{ESO}(1 / 1 / 5)$ at different temperatures 
Similar trends were observed in the degradation of PVC in the presence of mixed stabilizers $0.008 \mathrm{~g}$ of each metal carboxylate and 0.04 of ESO (Figures 15 and 16).

\subsubsection{Calculation of activation energy}

The degraded of PVC was carried out at different temperature in constant conditions (Figures 12, 13, 14). It was observed that the degradation of PVC increases with increasing temperature. The degradation rate was determined and the effect of temperature on the rate of dehydrochlorination are listed in Table 4. Then we may equalize the reaction rate to the degradation rate. Substitution of Arrhenius relation in general rate Equation (12) yields:

$\mathrm{R}=\mathrm{A}[\mathrm{PVC}]^{\alpha}[\mathrm{S}]^{\beta} \mathrm{e}^{-\mathrm{E}_{\mathrm{a}} / \mathrm{RT}}$

where $A, E_{a}$ and $T$ indicate collision parameter in Arrhenius equation, activation energy and absolute temperature, respectively. Other abbreviations have been defined already. In this manner, temperature is the sole variable. Recent Equation (13) may be rewritten as:

Table 4. Effect of temperature on the rate of dehydrochlorination of PVC with $0.5 \mathrm{~g}$ PVC

\begin{tabular}{|c|c|c|c|c|c|}
\hline \multirow{3}{*}{ Compound } & \multirow{2}{*}{\multicolumn{4}{|c|}{$\frac{\mathbf{R} \cdot 10^{2}[\text { conversion } \% / \mathrm{min}]}{\text { Temperature }\left[{ }^{\circ} \mathbf{C}\right]}$}} & \multirow{3}{*}{$\begin{array}{c}E_{a} \\
{\left[\mathrm{~kJ} \cdot \mathrm{mol}^{-1}\right]}\end{array}$} \\
\hline & & & & & \\
\hline & 170 & 180 & 190 & 200 & \\
\hline PVC & 4.1 & 4.8 & 6.3 & 8.0 & 38.6 \\
\hline $\mathrm{Hg} / \mathrm{Ca} / \mathrm{PVC}$ & 2.8 & 3.3 & 4.0 & 5.8 & 53.3 \\
\hline $\mathrm{Hg} / \mathrm{Ca} / \mathrm{ESO} / \mathrm{PVC}$ & 1.6 & 2.4 & 3.2 & 5.2 & 64.7 \\
\hline
\end{tabular}

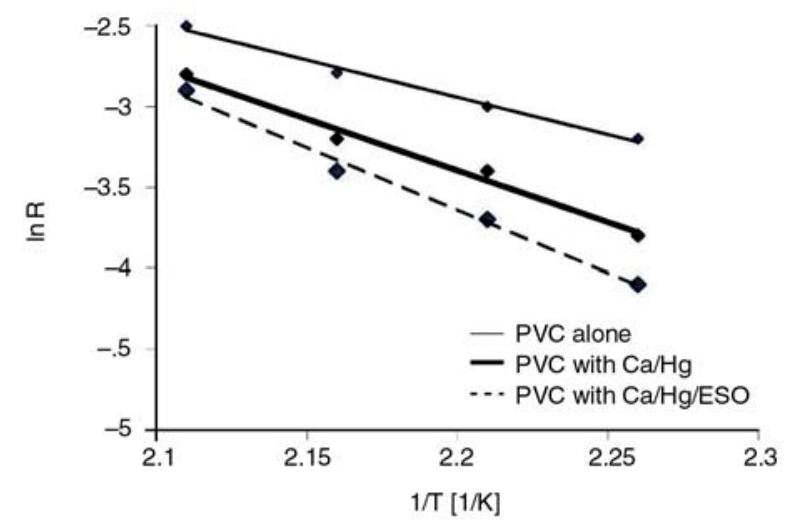

Figure 17. Plot of degradation rate upon the inverse of temperature in the absence and in the presence of mixture of stabilizers $\ln R=\ln k^{\prime}-\frac{E_{a}}{R T}$

where $k^{\prime}=A[\mathrm{PVC}]^{\alpha}[S]^{\beta}$. It means that if $\ln R$ versus $1 / T\left[\mathrm{~K}^{-1}\right]$ values are fitted with a straight line, the activation energies of reaction, slope of plot, may be derived. The results of Table 4 are depicted in Figure 17.

The data of Table 4 show that the variation of the rates and activation energies of dehydrochlorination of PVC. The degradation rates are lower and the activation energies are higher at the presence of stabilizers, compared to the absence of stabilizer. This daringly indicates that the polymer with stabilizer is more stable than the polymer alone.

Results observed in our laboratory are consistent with other investigative works [22].

\section{Conclusions}

In this work, we studied degradation and stability of PVC in the absence and presence of different stabilizers at various temperatures. Epoxidized sunflower oil shows excellent properties as a secondary stabilizer for PVC when used in combination with the synergetic metal soaps $(\mathrm{Hg} / \mathrm{Ca})$.

The marked effects of ESO on the thermal stabilization of PVC could not be observed by using without the synergetic metal soaps. This ternary system retards the development of dehydrochlorination and reduces the rate of degradation. In the first case, the synergism between ESO and metal soaps results from the reduction of the initial rate of DHC due to the reaction between $\mathrm{HCl}$ evolved at the early stages of DHC with ESO and metal soaps which reduces its catalytic effect on the degradation of PVC as well as etherification and esterification reactions of labile chlorine atoms leading to the formation of short polymer sequences which are responsible of the absence of initial coloration. Reduction of initial rate of DHC due to the reaction between $\mathrm{HCl}$ evolved at the early stages of dehydrochlorination with ESO and metal soaps which reduces its catalytic effect on the degradation of PVC. 


\section{References}

[1] Mohamed N. A., Al-Magribi W. M.: N-(Substituted phenyl) itaconimides as organic stabilizers for rigid poly(vinyl chloride) against thermal degradation. Polymer Degradation and Stability, 78, 149-165 (2002).

[2] Taghizadeh M. T., Fakhimi F.: Kinetics study of degradation and stabilizing effect of organic thermal stabilizers (EDTA. 1,2 propane diol, benzoic acid and phenol) for rigid poly(vinyl chloride). Iranian Polymer Journal, 14, 685-692 (2005).

[3] Sivalingam G., Madras G.: Effect of metal oxides/ chlorides on the thermal degradation of poly(vinyl chloride), poly(bisphenol a carbonate), and their blends. Industrial and Engineering Chemistry Research, 43, 7716-7722 (2004).

[4] Starnes W. H., Ge X.: Mechanism of autocatalysis in the thermal dehydrochlorination of poly(vinyl chloride). Macromolecules, 37, 352-359 (2004).

[5] Arkis E., Balköse D.: Thermal stabilization of poly(vinyl chloride) by organotin compounds. Polymer Degradation and Stability, 88, 46-51 (2005).

[6] Veronelli M., Mauro M., Bresadola S.: Influence of thermal dehydrochlorination on the photooxidation kinetics of PVC samples. Polymer Degradation and Stability, 66, 349-357 (1999).

[7] Andricic B., Kovacic T., Klaric I.: Kinetic analysis of the thermo-oxidative degradation of poly(vinyl chloride) in poly(vinyl chloride)/methyl methacrylatebutadiene-styrene blends 1 Isotermal degradation. Polymer Degradation and Stability, 78, 459-465 (2002).

[8] Mohamed N. A., Sabaa M. W., Yassin A. A.: Organic thermal stabilizers for rigid poly(vinyl chloride) IV. $\mathrm{N}$-aryl phthalimides. Polymer Degradation and Stability, 76, 355-365 (2002).

[9] Starnes W. H.: Structural and mechanistic aspects of the thermal degradation of poly(vinyl chloride). Progress in Polymer Science, 27, 2133-2170 (2002).

[10] Okieimen F. E., Eromonsele O. C.: Stabilizing effect of derivatives of Khaya seed oil on the thermal degradation of poly(vinyl chloride). European Polymer Journal, 36, 525-537 (2000).

[11] Minagawa M.: New developments in polymer stabilization. Polymer Degradation and Stability, 25, 121141(1989).

[12] Kalouskova R., Novotna M., Vymazal Z.: Investigation of thermal stabilization of poly(vinyl chloride) by lead stearate and its combination with synthetic hydrotalcite. Polymer Degradation and Stability, 85, 903909 (2004).

[13] Sabaa M. W., Mohamed R. R., Yassin A. A.: Organic thermal stabilizer for rigid poly(vinyl chloride) IX. NBenzoyl-N-p-substituted phenylthiourea derivatives. Polymer Degradation and Stability, 81, 431-440. (2003).
[14] Sabaa M. W., Mohamed N. A., Oraby E. H., Yassin A. A.: Organic thermal stabilizer for rigid poly(vinyl chloride) V. Benzimidazolylacetonitrile and some of its derivatives. Polymer Degradation and Stability, 76, 367-380 (2002).

[15] Stepek J., Daoust H.: Additives for plastics. New York, Springer-Verlag (1983).

[16] Wypych J.: On the mechanism of action of epoxy stabilizers in poly(vinyl chloride) compositions. Journal of Applied Polymer Science, 19, 3387-3389 (1975).

[17] Okieimen F. E., Sogbaike C. E.: Stabilising effect of derivatives of jatropha seed oil on the thermal degradation of in poly(vinyl chloride). European Polymer Journal, 12, 1457-1462 (1996).

[18] Anderson D. F., Mekenize D. A.: Mechanism of the thermal stabilization of poly(vinyl chloride) with metal carboxylates and epoxy plasticize. Journal of Polymer Science, 8, 2905-72922 (1970).

[19] Lida T., Kawato J., Maruyama K., Goto K.: Stabilization of poly(vinyl chloride).VII. synergisms between epoxy compounds and metal soaps. Journal of Applied Polymer Science, 34, 2355-2365 (1987).

[20] Benaniba M. T., Belhaneche-Bensemra N., Gelbard G.: Stabilizing effect of epoxidized sunflower oil on the thermal degradation of poly(vinyl chloride). Polymer Degradation and Stability, 74, 501-505 (2001).

[21] Okieimen F. E.: Studies in the utilization of deoxidized vegetable oils as thermal stabilizer for poly (vinyl chloride ). Industrial Crops and Products, 15, 71-75 (2002).

[22] Benaniba M. T., Belhaneche-Bensemra N., Gelbard G.: Stabilization of PVC by epoxidized sunflower oil in the presence of zinc and calcium stearates. Polymer Degradation and Stability, 82, 245-249 (2003).

[23] Rüsch M., Klaas G., Warwel S.: Complete and partial epoxidation of plant oils by lipase- catalyzed perhydrolysis. Industrial Crops and Products, 9, 125-132 (1999).

[24] Mohamed N. A., Sabaa M. W., Khalil Kh. D., Yassin A. A.: Organic thermal stabilizers for rigid poly(vinyl chloride) III. Contonal and cinnamal thiobarbituric acids. Polymer Degradation and Stability, 72, 53-61 (2001).

[25] Flory P. J.: Principles of polymer chemistry. Cornell University Press, Ithaca, (1953).

[26] Iván B., Kelen T., Tüdős F.: Degradation and stabilization of poly(vinyl chloride). in 'Degradation and Stabilization of Polymers' (Eds.: Jellinek H. H. G., Kachi H.) Elsevier, New York, 483-714 (1989).

[27] Tüdős F., Iván B., Kelen T., Kennedy J. P.: Degradation of virgin and modified chlorine containing polymers. in 'Developments in Polymer Degradation, Vol 6' (ed.: Grassie N.), Elsevier, London, 147-189 (1985).

[28] Iván B., Kennedy J. P., Kelen T., Tüdős F., Nagy T. T., Turcsanyi B.: Degradation of PVCs obtained by controlled chemical dehydrochlorination. Journal of Polymer Science: Polymer Chemistry Edition, 21, 2177-2188 (1983). 
[29] Iván B., Kennedy J. P., Kelen T., Tüdős F.: Cyclopentadienylation of PVC. Polymer Bulletin, 1, 415-420 (1979).

[30] Iván B., Kennedy J. P., Kelen T., Tüdős F.: Cyclopentadienylation of PVC,Characterization and thermal and thermooxidative degradation studies. Journal of Polymer Science. Polymer Chemistry Edition, 19, 925 (1981).

[31] Iván B., Kennedy J. P., Kelen T., Tüdős F.: Preparation, degradation, cyclopentadienylation, and grafting of PVCs containing relatively high levels of allylic chlorines. Journal of Macromolecular Science, Part A, 17, 1033-1043 (1982).

[32] Frye A. H., Horst R. W.: The mechanism of poly(vinyl chloride) stabilization by barium cadmium, and zinc carboxylates. I. Infrared studies. Journal of Polymer Science, 40, 419-439 (1959).

[33] Frye A. H., Horst R. W.: The mechanism of poly(vinyl chloride) stabilization by barium. cadmium, and zinc carboxylates. II. Radioactive studies. Journal of Polymer Science, 45, 1-12 (1960).
[34] Benaniba M. T., Belhanech-Bensemra N., Gelbard G.: Stabilizing effect of epoxidized sunflower oil on the thermal degradation of poly(vinyl chloride). Polymer Degradation and Stability, 74, 501-505 (2001).

[35] Abbas K. B., Laurence R. L.: Polyene sequence distribution in thermally degraded poly(vinyl chloride). Journal of Polymer Science, 13, 1889-1899 (1975).

[36] Beltran M., Marcilla A.: Fouried transform infrared spectroscopy applied to the study of PVC decomposition. European Polymer Journal, 7, 1135-1142 (1997).

[37] Mohamed N. A., Al-Afaleq E. I.: Aromatic 1,3,4-oxadiazoles as thermal stabilizers for rigid poly(vinyl chloride). Polymer, 40, 617-627 (1999).

[38] Shindo Y., Morukami M., Nishida T.: On the solution properties of chemically dehydrochlorinated poly (vinyl chloride). Journal of Polymer Science, Part B: Polymer Letters, 10, 555-560 (1972). 